\title{
Co-production helps ensure that new technology succeeds
}

\author{
Juliet Dobson digital content editor
}

The BMJ

Digital technology is a fact of modern life. With the NHS Long Term Plan embracing innovation and technology as a way to improve access to services, doctors will have to adapt to new ways of working. But while we all undergo the digital revolution we must not assume that new technology will work for everyone. Martin Roland is cautious (doi:10.1136/bmj.14713). Babylon's digital service, GP at Hand, provides medical care on a smartphone. A recent evaluation found that though the service met the needs of some patients it fell short elsewhere. Roland concludes that it "risks destabilising care for patients with the greatest needs."

Helen Salisbury, herself a GP, is equally sceptical (doi:10.1136/ bmj.14719). She isn't convinced that technology will reduce her workload. News that Amazon's Alexa will provide answers to patients' questions by using information from the NHS website leaves her questioning whether people really understand the nature and complexity of a GP's day to day work. Reflecting on a recent clinic she says, "I can't think of a single patient whose appointment would have been avoided by asking Alexa." New models of care using digital technology abound in the NHS. The NHS Near Me service, for example, offers online consultations to remote communities in Scotland, making specialist care more accessible (doi:10.1136/bmj.14770). The project was designed and developed jointly with patients but fell short of full "co-production," which would have required patients to be included in governance. The project found that patients preferred access at community clinics rather than at home. The team could also ensure that patients had suitable devices and technical skills to use the service.

Harlan Krumholz and Behnood Bikdeli (doi:10.1136/bmj.14686) look to digital solutions and co-production to help solve a problem highlighted by new research. For patients with a first episode of unprovoked venous thromboembolism, the risk of recurrence after stopping anticoagulant treatment is substantial (doi:10.1136/bmj.14363). Krumholz and Bikdeli suggest that having patients as partners in research could lead to personalised estimates of risk, helping patients make more informed decisions about their treatment.

Key to implementing any technology is understanding users and building systems they really want. Any technical fix must meet the needs of patients and doctors and must be properly tested to ensure it solves their most pressing problems. Co-production offers us one way to design services that actually work. 